[RADOCakbon, Vol. ]5, No. 1, 1973, P. 113-126]

\title{
UNIVERSITY OF KIEL RADIOCARBON MEASUREMENT'S VII
}

H. ERLENKEUSER and H. WILLKOMM

$\mathrm{C}^{14}$ Laboratory of the Institut für Kermphysik, University of Kiel

This list contains data obtained during 1971. Unless otherwise stated, all organic samples or organic fractions are carefully washed with dil. HCl and dil. NaOH to remove all carbonates. Age calculations are based on 95\% of NBS oxalic acid standard activity with modern value A.D. 1950. Results are calculated using the Libby half-life and are given in the B.P. scale. Also ages of shells are calculated with the NBS oxalic acid standard. Ages are not corrected for $\delta \mathrm{C}^{13}$ variations. Lrrors correspond to $I_{\sigma}$ variation of sample net counting rate including statistics of modern standard and background. Sample activities, if given in per cent refer to 0.95 NBS oxalic acid standard activity.

\section{ACKNOWLIDGMENTS}

We thank Heidi Metmer for measuring $\delta C^{1: 3}$ values and Günter Enge for treating water samples. We are indebted to the Deutsche Forschungsgemeinschaft for financial support within the Sonderforschungsbereich (joined research program) 95-Interactions water sea-floor boundary.

I. GEOLOGIG SAMPLAS

A. Terrestrial Samples

\section{KI-330. Recent twigs, 1969}

$156.3 \pm 0.8 \%$

$\delta\left(i^{\prime \prime}=-25.9 \%\right.$

Twigs (Malnus domeslicus) grown summer 1969 near Kiel (54 18.5' N Lat, $10^{\circ} 4.3^{\prime} \mathrm{E}$ Long), Germany. Coll. Sept. 1969.

\section{Esterweger Dose, peat development}

Peat from Esterweger Dose (53 $3.1^{\prime} \mathrm{N}$ Lat, $7^{\circ} 37.1^{\prime} \mathrm{E}$ Long), emerged bog near Papenburg, NW Germany. Coll. 1969 and subm. by K. Müller, Bot. Inst., Univ. Kiel. Comment: samples complete series in R., 1971 , v. 13, p. 395.

\section{KI-337. Light peat, 65 to $67 \mathrm{~cm}$ depth \\ Underlies K I-396.}

$16.50 \pm 50$

$$
\begin{gathered}
\text { A.D. 300 } \\
\delta C^{13}=-25.3 \%
\end{gathered}
$$

\section{KI-314. Light peat, $10 \mathrm{~cm}$ depth}

$100.2 \pm 0.5 \%$

Peat (Sphagmum cuspidalum) with twios of Ficuceae and Sohemen zeria.

\section{KI-516.01. Leopold oak, tree-ring 90 to 95}

$$
\begin{gathered}
\mathbf{3 5 5 0} \mathbf{5 5 5} \\
\mathbf{1 6 0 0} \text { B.c. } \\
\delta C^{13}--25.0 \%
\end{gathered}
$$

Oak with 380 rings, from excavations at center of Kiel (54 $19.4^{\prime}$ $\mathrm{N}$ Lat, $10^{\circ} 8.5^{\prime} \mathrm{E}$ Iong), Germany, Coll. $197 \mathrm{l}$ and subm. by F. R. 
Averdieck, Inst. f. Ur-und Frïhgeschichte, Univ. Kiel. Comment: tree is for dendrochronologic study of Schleswig-I Iolstein.

\section{Mammoth tusk, Tettenhausen}

Part of mammoth tusk, from gravel pit near Iettenhausen (470 57.6' N Lat, $12^{\circ} 45.7^{\prime} \mathrm{E}$ Long), Bavaria, Germany. Coll. 1969 by Jolannes Seidel, 8221 Reichersclorf, Post Petting; subm. 1970 by Edith Ebers, 8121 Haunshofen. Comment (J.S. and E.L.): tusk was covered by 460 (m gravel ("I atufenschotter") and lay $580 \mathrm{~cm}$ underground. Contamination by lime is possible. Comment: sample is divided into pieces of different purity (visual criteria), yet age of any fraction is much lower: than expected. Organic parts were too small to measure.

KI-358.031.

$$
\begin{array}{r}
3620 \pm 70 \\
1670 \mathrm{B.c} . \\
\delta C^{13}=-9.9 \% \text {. }
\end{array}
$$

Selected, purely white material, surface mechanically removed.

KI-358.041.

$4030 \pm 80$

2080 B.C.

Fragments like .031, but with small dark impurities.

KI-358.051.

Remains of .031 and .041, darkened surface parts.

\section{KI-457. Boxberg, 1971}

Soil containing mixture of fossil $P_{x^{-}}, B_{1^{-}}$, and $A_{h_{1}}$-horimons 80 to $100 \mathrm{~cm}$ under ground from slope of Boxberg $\left(5.1^{\circ} 4.3^{\prime}>\mathrm{Lat}, 9^{\circ} 45.0^{\prime} \mathrm{E}\right.$ Long), Schleswig-Holsten, Germany. Coll. 1971 and subm. by G. M. Stanschus, Gcog. Inst., Univ. Kiel. Comments (G.M.S.): horizons are residues of solifluctions during last interglacial or late glacial. Measured $C^{1+1}$ content indicates recent contamination. Rootlets were washed away carefully. Carbonates were removed in usual way by diluted HCl.

\section{B. Lakes and Underground Water}

\section{Plöner See, recent water and plants}

Series is continued from 1970 (cf. R., 1971, v. 13, p. 329).

\section{KI-324. Potamogeton perfoliatus, Plïn $1969 \begin{gathered}122.7 \pm 0.8 \% \\ \delta C^{1.3}=-18.7 \%\end{gathered}$}

Aquatic plants ca. 50 to $100 \mathrm{~cm}$ under surface of Grosser Plöner See (510 9.0 N Lat, $10^{\circ} 25.1 \mathrm{E}$ Long), NW Germany. Coll. Sept. 1969 and subm. by F. R. Averdick. 
TABLE 1

$\mathrm{C}^{14}$ dates of Plöner See sediments

\begin{tabular}{|c|c|c|c|c|c|c|}
\hline \multirow[b]{3}{*}{$\begin{array}{l}\text { Lab. } \\
\text { no. }\end{array}$} & \multirow{3}{*}{$\begin{array}{c}\text { Kiel Cit Lab. } \\
\text { Depth } \\
\text { within } \\
\text { sediment } \\
\text { (cm) }\end{array}$} & \multicolumn{3}{|c|}{ Grosser Plöner See } & \multicolumn{2}{|c|}{$\mathrm{KI}-85$ and $\mathrm{KI}-246$} \\
\hline & & \multicolumn{2}{|c|}{ Organic fraction } & \multicolumn{2}{|c|}{ Inorganic fraction } & \multirow{2}{*}{$\begin{array}{l}\text { Differ- } \\
\text { ence } \\
\text { ol Libby } \\
\text { ages (yr) }\end{array}$} \\
\hline & & $\begin{array}{l}\text { Libby age } \\
\pm 1 \sigma \text { в.P. }\end{array}$ & $\begin{array}{c}\delta \mathrm{C}^{13} \\
\%\end{array}$ & $\begin{array}{l}\text { Libby age } \\
\pm \text { lo B.P. }\end{array}$ & $\begin{array}{c}\delta \mathrm{C}^{13} \\
\%(t o\end{array}$ & \\
\hline 5.20 & 550 to 580 & $1620 \pm 65$ & -29.7 & $3160 \pm 90$ & -1.7 & 1540 \\
\hline 85.21 & 580 to 608 & $1540 \pm 80$ & -29.2 & $3260 \pm 75$ & -1.4 & 1720 \\
\hline 85.22 & 608 to 634 & $1700 \pm 80$ & -30.6 & $3800 \pm 65$ & -0.9 & 2100 \\
\hline 85.23 & 650 to 680 & $1700 \pm 90$ & -30.6 & $4230 \pm 140$ & -1.4 & 2530 \\
\hline 85.24 & 680 to 710 & $2160 \pm 110$ & -32.2 & $3860 \pm 110$ & -0.4 & 1700 \\
\hline
\end{tabular}

\section{KI-487. Aquatic plants, Plön 1971}

\section{KI-486. Surface water, Plïn 1971}

$120.9 \pm 0.8 \%$

$=-3.8 \%$ N Lat, $10^{\circ} 25.1^{\prime}$ E Lono). Well

\section{KI-485. Depth water, Plïn 1971}

$121.1 \pm 0.7 \%$

Pumped from $26.5 \mathrm{~m}$ under surface at same position as KI-486.

\section{Pliiner See series}

Lake sediments of the Grosser Plöner See (54 $5.5^{\circ} \mathrm{N}$ Lat, $10^{\circ} 25.1^{\prime}$ E Long), NW Germany. Coll. and subm. 1966 by F. R. Averdieck and W. Ohle, Max-Planck-Inst. f. Limnol., Plön. Continuation of former dates (R., 1971, v. 13, p. 397). Dates are given in Table 1. Comment: results confirm large difference between organic and inorganic fraction in 6 to $7 \mathrm{~m}$ depth.

\section{Ravensherg series}

Lake scdiments under small emerged bog near Südcrlügum (54º $50.7^{\prime} \mathrm{N}$ Lat, $8^{\circ} 57.5^{\prime}$ E Long), NW Schleswig-Holstein, Germany. Coll. 1970 and subm. 1971 by Hartmut Usinger, Bot. Inst., Univ. Kiel, who also made pollen analysis.

\section{KI-419. RB 12.14}

$$
\begin{aligned}
& 12,080 \pm 110 \\
& 10,130 \text { B.C. }
\end{aligned}
$$

Gyttja $462 \mathrm{~cm}$ under water surface. Comment (H.U.): expected age: some centuries before Bölling, ca. 11,400 B.c. 
KI-420. RB 20-22

$12,650 \pm 280$

10,700 в.C.

$\delta C^{13}=-23.9 \%$ Bölling.

Gyttja $446 \mathrm{~cm}$ under water surface. Comment (H.U.): middle of

\section{KI-421. RB 28-30}

$10,840 \pm 130$

8890 B.C.

$\delta C^{13}=-20.5 \%$

Gyttja $430 \mathrm{~cm}$ under water surface. Comment (H.U.): beginning of Alleröd. $\mathrm{C}^{14}$ age ca. 1100 yr too young.

\section{Well series, Kiel 1971}

Water samples of clifferent wells near Kiel, Germany, coll. Sept. 1971 by G. Enge, Inst. $f$. Kernphysik, Univ. Kiel. $\mathrm{CO}_{2}$ of $50 \mathrm{~L}$ acidified water was liberated on stirring by bubbling nitrogen and frozen out with liquid air (Enge, 1971).

\section{KI-475. Brunnen der Universität}

$55.1 \pm 0.4 \%$

38 to $65 \mathrm{~m}$ deep (54 $22.3^{\prime} \mathrm{N}$ Lat, $10^{\circ} 7.3^{\prime} \mathrm{E}$ Long).

$$
\delta C^{13}=-14.4 \%
$$

$$
\begin{array}{lr}
\text { KI-476. Brunnen 11, Schulensee } & \mathbf{5 2 . 8} \pm \mathbf{0 . 4 \%} \\
78 \text { to } 138 \mathrm{~m} \text { deep }\left(54^{\circ} 17.6^{\prime} \mathrm{N} \text { Lat, } 10^{\circ} 6.1^{\prime}\right. \text { E Long). } & \delta C^{1.3}=-13.3 \%
\end{array}
$$

\section{KI-477. Brunnen 5, Kiel-Wik}

149 to $199 \mathrm{~m}$ deep $\left(54^{\circ} 20.7^{\prime} \mathrm{N}\right.$ Lat, $10^{\circ} 7.1^{\prime} \mathrm{E}$ Long).

\section{Kiel Bay Sediment}

\section{Marine Samples}

Muddy sediment from NW Kiel Bay $\left(54^{\circ} 46.3^{\prime} \mathrm{N}\right.$ Lat, $10^{\circ} 11.3^{\prime} \mathrm{E}$ Long), W Baltic Sea. Coll. and subm. by F. Werner, Geol. Inst., Univ. Kiel, and H. Erlenkeuser and H. Willkomm, March 1971. Sample is from surface sediment, at $-26 \mathrm{~m}$, with Box Corer (cross section $20 \times 28$ $\mathrm{cm}$ ). Sediment core was cut into slices $1 \mathrm{~cm}$ thick parallel to sediment surface. Samples were boiled with $1 \% \mathrm{HCl}$ to remove traces of carbonate, filtered, washed to neutrality, and dricd. Samples were converted to $\mathrm{CO}_{2}$ by dry combustion. Results are given in Table 2. Comment: sediment core is from area with high sedimentation rate (ca. . $\mathrm{mm}$ /year) with postglacial mud layers up to $20 \mathrm{~m}$ thick (Seibold $e t$ al., 1971). Radiographic methods show a layered structure of sediment core between 0 to $90 \mathrm{~mm}$ depth, whereas below $160 \mathrm{~mm}$ sediment is homogeneous without recognizable structure. Measurements give an apparent. age of pre-bomb sediments of ca. $1000 \mathrm{yr}$. Steep increase of $\mathrm{C}^{14}$ activity between at least $68 \mathrm{~mm}$ and surface is probably due to bomb-produced $\mathrm{C}^{14}$. Significance of the older layer interposed at 100 to $70 \mathrm{~mm}$ is still under discussion. 
Kiel Bay Macrobenthic series, 1971

Shells of live mollusks at diflerent positions in W Kiel Bay. Coll. by $A$. Fritsche, R. S. Newton, F. Werner, Geol. Inst., Univ. Kiel, and H. Willkomm, March 1971; subm. by H. Erlenkeuser and H. Willkomm. Only carbonate fraction was dated. Large specimen $($ diam. $>5 \mathrm{~cm})$ was used.

\section{KI-407.011. Aero S.W., 11777}

$129.5 \pm 1.2 \%$

Cyprina islandica, $\left(54^{\circ} 46^{\circ}\right)^{\prime} \mathrm{N}$ Iat, $10^{\circ} 1153^{2}$ gray muddy sediment surface, $26 \mathrm{~m}$ water depth.

\section{KI-408.011. Aero S.W., 11779}

$105.8 \pm 0.8 \%$

Cyprina islandica, $\left(54^{\circ} 47.0^{\prime} \mathrm{N}\right.$ Lat, $10^{\circ} 14.6^{\prime} \mathrm{E}$ Long), from blackgray muddy sediment surface, $29 \mathrm{~m}$ water depth.

\section{Table 2}

$\mathrm{C}^{14}$ dates of Kiel Bay sediments

Age calculations are made without $\delta \mathrm{C}^{13}$ corrections

\begin{tabular}{|c|c|c|c|}
\hline \multirow[t]{3}{*}{ Kiel C ${ }^{14}$ Lab. } & \multicolumn{2}{|c|}{$\begin{array}{l}\text { Kicl Bay sediments } \\
\text { Aero S.W., } 11777-3\end{array}$} & {$[-483$} \\
\hline & $\begin{array}{l}\text { Depth } \\
\text { within }\end{array}$ & \multicolumn{2}{|c|}{ Organic fraction } \\
\hline & $\begin{array}{l}\text { sediment } \\
(\mathrm{mm})\end{array}$ & $\begin{array}{l}\text { Libby age } \\
\pm 1 \text { o в.P. }\end{array}$ & $\begin{array}{l}\delta C^{13} \\
(\% / i)\end{array}$ \\
\hline 483.01 & 0 to 20 & $540 \pm 45$ & -23.3 \\
\hline 483.02 & 20 to 32 & $450 \pm 60$ & -22.5 \\
\hline 483.03 & 32 to 44 & $680 \pm 55$ & -22.8 \\
\hline 483.04 & 44 to 56 & $955 \pm 55$ & -22.5 \\
\hline 483.05 & 56 to 68 & $900 \pm 40$ & -21.9 \\
\hline 483.06 & 68 to 80 & $1060 \pm 85$ & -21.6 \\
\hline 483.07 & 80 to 92 & $1625 \pm 65$ & -21.6 \\
\hline 483.08 & 92 to 104 & $1480 \pm 60$ & -21.1 \\
\hline 483.09 & 104 to 116 & $1475 \pm 70$ & -21.3 \\
\hline 483.10 & 116 to 128 & $1065 \pm 60$ & -21.5 \\
\hline 483.11 & 128 to 140 & $1325 \pm 45$ & -21.1 \\
\hline 483.12 & 140 to 152 & $1095 \pm 80$ & -22.3 \\
\hline 483.13 & 152 to 164 & $1120 \pm 45$ & -20.1 \\
\hline 483.14 & 164 to 188 & $1110 \pm 55$ & -20.1 \\
\hline 483.15 & 188 to 212 & $1055 \pm 55$ & -20.5 \\
\hline 483.16 & 212 to 236 & $920 \pm 50$ & -20.3 \\
\hline 483.17 & 236 to 260 & $810 \pm 60$ & -20.7 \\
\hline 483.18 & 260 to 284 & - & -- \\
\hline 483.19 & 284 to 308 & $750 \pm 55$ & -20.0 \\
\hline 483.20 & 308 to 332 & $940 \pm 60$ & -21.5 \\
\hline
\end{tabular}




\section{KI-410.011. Aero S.W., 11818}

$103.4 \pm 0.5 \%$ grained sand bottom, $16 \mathrm{~m}$ water depth.

\section{KI-411.011. Aero S.W., 11849}

$113.5 \pm 0.4 \%$

Cyprina islandica, $\left(54^{\circ} 46.9^{\prime} \mathrm{N}\right.$ Lat, $10^{\circ} 5.4^{\prime} \mathrm{E}$ Long) from fine grained sand bottom, $19 \mathrm{~m}$ water depth.

\section{KI-412.011. Aero S.W., 11851}

$103.0 \pm 0.8 \%$

Cyprina islandica $\left(54^{\circ} 46.9^{\prime} \mathrm{N}\right.$ Lat, $10^{\circ} 5.8^{\prime} \mathrm{E}$ Long), from brown muddy sand bottom, $23 \mathrm{~m}$ water depth.

\section{KI-413.011. Aero S.W., 11857}

$104.0 \pm 0.5 \%$

Big Cyprina islandica $(8$ Long) from gray muddy sand bottom, $13.5 \mathrm{~m}$ water depth.

\section{KI-415.011. Aero S.W., 11859}

$106.7 \pm 0.6 \%$

Cyprina islandica, $\left(54^{\circ} 43.3^{\prime} \mathrm{N}\right.$ Lat, $10^{\circ} 5.7^{\prime} \mathrm{E}$ Long $)$ from coarse grained sand bottom, $13 \mathrm{~m}$ water depth. Comment: shells show considerably low $\mathrm{C}^{14}$ activity compared with recent macrobenthic samples KI-414, $-524,-528$ and -529 , even in view of life-span of mollusks, i.c., ca. 10 yr (Kühlmorgen-Hille, 1963).

\section{KI-414. Aero S.W., 11858}

$133.2 \pm 0.9 \%$ $\delta C^{1 . i}=-23.0 \%$

Laminaria digitata, $\left(54^{\circ} 47.5^{\prime} \mathrm{N}\right.$ Lat, $10^{\circ} 4.0^{\prime} \mathrm{E}$ Long) growing on lag sediment, $10 \mathrm{~m}$ water depth. Coll. and subm. by R. S. Newton and F. Werner, Geol. Inst., Univ. Kiel, and H. Erlenkeuser and H. Willkomm, March 1971.

\section{Stoller Grund}

Samples from 'Stoller Grund', (54 $31.8^{\prime} \mathrm{N}$ Lat, $10^{\circ} 10.7^{\prime} \mathrm{E}$ Long) SW Kiel Bay. Grown on lag sediment. Coll. and subm. by II. Frlenkeuser, Nov. 1971.

KI-524. Stoller Grund, 1

Red algae assoc.

KI-525. Stoller Grund, 2 Red and brown algae assoc.

\section{Stoller Rinne}

Varied macrobenthic samples from 'Stoller Rinne' $\left(54^{\circ} 29.5^{\prime} \mathrm{N}\right.$ Lat, $10^{\circ} 11.5^{\prime}$ E Long) SW Kiel Bay. Surface sediment is lag on till and
$132.4 \pm 0.8 \%$ $\delta C^{1,3}=-24.7 \%$

$126.6 \pm 2.6 \%$ $\delta C^{13}=-28.8 \%$ 
sand. Water depth ca. $10 \mathrm{~m}$. Coll. and subm. by H. Erlcnkeuser, Nov 1971 .

\section{KI-528.01. Stoller Rinne, 1}

Small specinen (Astropecten irregularis) diam. $<1 \mathrm{~cm}$.

KI-528.02. Stoller Rinne, 2

Brcad-crumb sponges (Inalichondria panicea Pall.)

\section{KI-528.03. Stoller Rinne, 3}

Red algale.

\section{KI-528.04. Stoller Rinne, 4}

Brown algae. $\begin{aligned} & 134.9 \pm 1.2 \% \\ & \delta C^{13}=-19.1 \%\end{aligned}$

$132.5 \pm 0.7 \%$ $\delta C^{13}=-27.3 \%$

$130.8 \pm 0.9 \%$ $\delta C^{1.3}=-31.8 \%$

$133.6 \pm 0.7 \%$ $\delta C^{13}=-25.3 \%$

General Comment: if corrected for isotopic fractionation, sample activities do not show significant differences and are higher than water activity (KI-531).

\section{Schilksee Bay}

Varied macrobenthic samples from 'Grashügel' (54⒉3' N Lat, 10' $11.7^{\prime} \mathrm{E}$ Long), submarine hillock in Schiiksce Bay in outer Kiel Fjord, W Baltic Sca. Lagg and sand sedinent surface, 5 to $12 \mathrm{~m}$ water depth. Coll. and subm. by H. Erlenkcuser, Nov. 1971.

\section{KI-529.01. Schilksee, I}

$129.1 \pm 1.4 \%$

Bread-crumb sponge (Halichondria panicea Pall.)

$$
\delta C^{13}=-24.9 \%
$$

\section{KI-529.02. Schilksee, 2}

$124.3 \pm 2.0 \%$

Small specimen (Astropecten irregularis) diam. $<\mathbf{l} \mathrm{cm}$.

$$
\delta C^{13}=-18.9 \%
$$

\section{KI-529.03. Schilksee, 3}

$128.4 \pm 1.1 \%$

Red algac.

$\delta C^{13}=-27.5 \%$

General Comment: activity of K I-529 samples is significantly Jower than open sea sample activity (K I-524, -525, and -528).

\section{KI-531. Schïnberger Strand}

$$
126.9 \pm 0.7 \%
$$
water $\left(54^{\circ} 25.0^{\prime} \mathrm{N}\right.$ Lilt, $10^{\circ} 25.0^{\prime} \mathrm{E}$ Long), S Kiel Bay, CO. liberated from inorganic cabonates. Coll. and subm. by H. Erlenkeuser, Dec. 1971. Water was taken at head of groin built into the open sea, $50 \mathrm{~m}$ apart from strand. Water depth $5 \mathrm{~m}$, sand sediment surface. ComInenl: activity corrected for isctopic fractionation seems too low compared to benthonic samples KI-114, -524, $-525,-528$, and -529 . 


\section{Landsort Basin}

Muddy sediments from Landsort. Basin $\left(58^{\circ} 40^{\prime} \mathrm{N}\right.$ Lat, $18^{\circ} 20^{\prime} \mathrm{E}$ Long), middle Baltic Sea. Coll. and subm. 1971 by E. Suess, Geol. Inst., Univ. Kiel. Sediment surface, $-459 \mathrm{~m}$. Samples are from box core $9 \mathrm{~m}$ long. Depths refer to sediment surface.

\section{KI-405.020. 150 to $190 \mathrm{~cm}$ depth \\ $1700 \pm 110$ A.D. 250 \\ $\delta C^{13}=-26.8 \%$}

Sample is treated with hot $1 \% \mathrm{HCI}$, filtered, washed, and dried before combustion. Final content of $\mathrm{C}_{\mathrm{org}}$ is $1.4 \%$.

\section{KI-405.022. 180 to $190 \mathrm{~cm}$ depth}

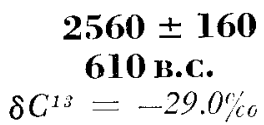

Same material as KI-405.020. Treated with hot $1 \%$ HCl, filtered and washed. Additionally boiled with $1 \% \mathrm{NaOH}$, washed to neutrality by centrifuging and decanting. Final $\mathrm{C}_{\mathrm{org}}$ content is $0.8 \%$. Comment: as indicated by low $\delta \mathrm{C}^{13}$ value, KI-405.022 mainly consists of organic complexes that have become highly resistant to chemical attack during their history of diagenesis (Degens, 1969; Williams et al., 1970).

\section{KI-405.030. 340 to $348 \mathrm{~cm}$ depth}

$1770 \pm 85$

A.D. 180

$$
\delta C^{13}=-26.2 \%
$$

Sample treated with $1 \%$ HCl only. Final $\mathrm{C}_{\text {org }}$ content $1.6 \%$.

\section{KI-405.040. 366 to $376 \mathrm{~cm}$ depth}

$$
\begin{gathered}
11,030 \pm 170 \\
9080 \text { в.C. }
\end{gathered}
$$

Sample dried only. $\mathrm{C}_{\text {or }}$ content is $4.5 \%$. Comment: sample might be contaminated with inorganic carbonate as guessed from underlaying samples (undated). Correction for inorganic carbonate aided by $\delta \mathrm{C}^{1.3}$ value reduces $\mathrm{C}^{14}$ age to ca. $8300 \mathrm{yr}$ B.r.; still too old in view of deeper samples.

\section{KI-405.05. 436 to $450 \mathrm{~cm}$ depth} $\mathrm{mMol} / \mathrm{g}$.

No chemical pretreatment. $\mathrm{C}_{\text {org }}$ content $2.0 \%$. Carbonates ca. 0.10

KI-405.05-20323.

$3410 \pm 95$

First measurement of sample.

1460 B.c.

$$
\delta C^{13}=-27.9 \%
$$

KI-405.05-20340.

Second measurement. Comment: same counting gas as KI-405.05- 
20323, but purificd a 2nd time. Gas was purified because lst sample mass spectrum showed unusual mass lines due to unknown contaminants. Weighted mean $\mathrm{C}^{14}$ age: $3140 \pm 85$ в.P.

\section{KI-405.06. 633 to $640 \mathrm{~cm} \mathrm{depth}$}

No chemical pretreatment. $\mathrm{C}_{\text {org }}$ content $1.5 \%$. Carbonates ca. 0.12 $\mathrm{mMol} / \mathrm{g}$. Comment: mass spectrum contaminated. $\delta \mathrm{C}^{13}$ might be too low (sce KI-405.05).

\section{KI-405.07. 880 to $890 \mathrm{~cm}$ depth}

$$
\begin{aligned}
& 4810 \pm 95 \\
& 2860 \text { B.c. } \\
& \delta C^{13}=-29.2 \%
\end{aligned}
$$

No chemical pretreatment. $\mathrm{C}_{\text {org }}$ content $1.2 \%$. Carbonates ca. 0.02 $\mathrm{mMol} / \mathrm{g}$. Comment: mass spectrum contaminated. $\delta \mathrm{C}^{13}$ value might be too low (see KI-405.05).

General Comment: samples dated for chemical and microbiological studies on development of middle Baltic Sea (Suess, 1971).

\section{Canary Islands}

Mollusk shells from littoral terraces of diflerent heights above sea level for dating ancient landlift of Canaries. Coll. 1965 and subm. 1970 by Heinz Klug, Geog. Inst., Univ. Kiel (Klug, 1968).

\section{KI-359. Gran Canaria, Bañaderos, $65 \mathrm{~m}$}

$$
\begin{array}{r}
>\mathbf{3 7 , 9 0 0} \\
(<\mathbf{0 . 8 9 \% )} \\
\delta C^{13}=-1.2 \% \%
\end{array}
$$

Shells from Gran Canaria (28 $38.5^{\prime} \mathrm{N}$ Lat, $15^{\circ} 32^{\prime} \mathrm{W}$ Long) at $+65 \mathrm{~m}, 5$ to $90 \mathrm{~cm}$ below surface. Statistical certainty of age estimate is $90 \%$.

\section{KI-360. Gran Canaria, Arucas, $25 \mathrm{~m}$}

$$
\begin{array}{r}
>38,800 \\
(<0.80 \%)
\end{array}
$$

Shells from Gran Canaria (28 37.3' N Lat, $15^{\circ} 31^{\prime} \mathrm{W}$ Long) from terrace at $+25 \mathrm{~m}, 5$ to $25 \mathrm{~cm}$ below surface. Statistical certainty of age estimate is $90 \%$.

\section{KI-361. Fuerteventura, Jandia, $55 \mathrm{~m}$}

Different shells belonging to a terrace, $+55 \mathrm{~m}$, at Fuerteventura $\left(28^{\circ} 3^{\prime} \mathrm{N}\right.$ Lat, $14^{\circ} 22^{\prime} \mathrm{W}$ Long). The 3 largest shells were treated separately, and their carbonate gave following ages (activity in $\%$ of modern in brackets).$$
(0.58 \pm 0.22 \%)
$$$$
41,300
$$$$
-4000
$$
KI-361.011.$$
\delta C^{15}=-0.5 \%
$$$$
\text { 39,300 в.с. }
$$ 
KI-361.021.

$$
(2.49 \pm 0.46 \%)
$$

$$
\begin{array}{r}
\delta C^{1.3}=-3.1 \% \\
(\mathbf{4 . 6 1} \pm \mathbf{0 . 2 4 \%}) \\
\delta C^{1.3}=-0.9 \%
\end{array}
$$$$
27,700 \text { B.C. }
$$

KI-361.031.

$24,720 \pm 420$

22,770 B.C.

Comment: according to different activity, at least the 2 older shclls must have been redeposited.

\section{KI-362. Lanzarote, Montaña Roja, 6 m

$$
(5.91 \pm 0.40 \%)
$$

$22,730 \pm 550$

Pupura hacmastoma L. from Lanzarote $\left(28^{\circ} 51.5^{\prime} \mathrm{N} \mathrm{I}_{a} a t, 13^{\circ} 5 \mathrm{I}^{\prime}\right.$ W Long), at $+6 \mathrm{~m}$.

KI-363. Lanzarote, Los Ajaches, $55 \mathrm{~m}$

$$
37,300+4000
$$

35,300 в.C.

$$
(0.21 \pm 0.08 \%)
$$

$\delta C^{13}=-2.6 \%$

I'ermetus Lumbicatis L., from Lanzarote $\left(28^{\circ} 51.5^{\prime} \mathrm{N}\right.$ Lat, $13^{\circ}$ 46.3' W Long) from terrace at $+55 \mathrm{~m}$.

KI-364. Gran Canaria, Agaete Nord

Shells from Gran Cimaria (28 $36^{\circ} \times$ Lat, $15^{\circ} 41^{\prime} \mathrm{W}$ Long) from terrace at +85 to $+91 \mathrm{~m}$.

KI-365. Fuerteventura, Los Malinos, 6 m

$$
(0.88 \pm 0.35 \%)
$$

Shells (Cassis sp.) from W Fuerteventura $\left(28^{\circ} 31^{\prime} \mathrm{N}\right.$ Lat, $14^{\circ} 4^{\prime}$ W Long).

KI-501. Lanzarote, Montaña Roja, 6 m

Patella intermedia, from same position as KI-362. Statistical certainty of age estimate is $90 \%$.

\section{Probstei coastline series}

Peat and wood from Baltic sea const of Probstei, Schleswig-Holstein, indicating geomorphologic development of coastline.

\section{KI-80. Schön 1}

$$
\begin{aligned}
& 1170 \pm 100 \\
& \text { A.D. } 780 \\
& \delta C^{\prime 3}=-28.5 \%
\end{aligned}
$$

Root of tree from peat covered with ca. $80 \mathrm{~cm}$ sand and gravel at Schönberg beach $\left(54^{\circ} 24.8^{\prime} \mathrm{N}\right.$ Lat, $10^{\circ} 25.3^{\prime}$ E Long). Coll. 1966 and subm. by H. Willkomm. 
KI-220. Schön 2

Root of tree $30 \mathrm{~m}$ behind Schönberg beach $\left(54^{\circ} 25.7^{\prime} \mathrm{N}\right.$ Lat, $10^{\circ}$ $22.7^{\prime} \mathrm{E}$ Long). Sample lay 80 to $90 \mathrm{~cm}$ underground in peat covered by $50 \mathrm{~cm}$ sea sand indicating later transgression. Coll. 1967 and subm. by H. Willkomm.

\section{KI-366. Köhler 2}

Peat from surface of sea floor, ca. $30 \mathrm{~m}$ from shore $\left(54^{\circ} 25.7^{\prime} \mathrm{N}\right.$ Lat, $10^{\circ} 23.3^{\prime}$ E Long). Coll. 1969 and subm. 1970 by Jürgen Köhler, (icog. Inst., Univ. Kiel.

\section{KI-367. K̈̈hler 1}

$$
\begin{aligned}
& 3080 \pm 65 \\
& 1130 \text { B.c. } \\
& \delta C^{13}=-28.4 \%
\end{aligned}
$$

Wood from peat covered with sind at sea floor near Stakendorf

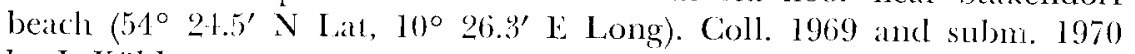
by J. Köhler.

\section{KI-381. 10056, 200 to $220 \mathrm{~cm}$ under sea floor}

$$
\begin{gathered}
11,880 \pm 100 \\
9930 \text { B.C. } \\
\delta C^{t .2}=-2 s .1 \% c t
\end{gathered}
$$

Peat from bottom of Baltic Sea ( $54^{\circ} 34.24^{\prime} \mathrm{N}$ Lat, $\left.10^{\circ} 6.21^{\prime} \mathrm{E} \mathrm{Lomg}\right)$, water depth 29 m. Coll. 1969 and subm. 1970 by F. Werner, Gcol. Inst., Univ. Kicl. Comment: continues Kieler Bucht series (R., 1971, v. 13, p. 3341$)$.

\section{I. ARCHAEOLOGIC: SAMIPLFS}

\section{KI-82. Burg Ahr}

$$
\begin{aligned}
& 490 \pm 40 \\
& \text { A.D. } 1460 \\
& \delta C^{1}=-21.1 \%
\end{aligned}
$$

Cut of ceiling rafter of Kencnate (ladies bower) of Ahr castle near Altenahr/Eifel $\left(50^{\circ} 30^{\prime} \mathrm{N}\right.$ Lat, $6^{\circ} 59^{\prime}$ E Long), W Germany. Coll. 1966 and subm. by $\mathrm{H}$. Erlenkeuscr. Comment: it is really as old as the guide tells.

\section{Alt-Liibeck}

Plant residues under wooden foundation of mound of Slovanic castle Alt-Lübeck (53 $55^{\prime} \mathrm{N}$ Lat, $10^{\circ} 43^{\prime} \mathrm{E}$ Long), ca. $5 \mathrm{~km} \mathrm{~N}$ of present Lübeck, Germany. Coll. 1968 and subm. 1969 by F. R. Averdieck.

\section{KI-278. Sambucus twigs}




$$
\begin{array}{r}
1100 \pm 45 \\
\text { A.D. 850 } \\
\delta C^{13}=-28.3 \%
\end{array}
$$

KI-289. Peat

Comment (F.R.A.): very local character of mixture of swamp and ruderal flora makes palynologic zonation difficult. Expected age of wood and peat is 11 th century or later.

\section{Haithabu}

Wood from Haithabu $\left(54^{\circ} 30^{\prime} \mathrm{N}\right.$ Lat, $9^{\circ} 33^{\prime} \mathrm{E}$ Long), ancient Viking town near Schleswig, NW Germany. Coll. by Josef Bauch and Dieter Eckstein, Ordinariat f. Holzbiol., Hamburg 80; subm. 1967 by F. R. Averdieck. Comment (J.B. and D.E.): samples are connected to dendrochronology of Haithabu, which is not yet connected to present. Tree rings indicate KI-242 is 280 yr older than KI-24l. Measured $\mathrm{C}^{1-4}$ ages are ca. $100 \mathrm{yr}$ older than expected.

\section{KI-241. Haithabu, Probe 4}

\section{KI-242. Haithabu, Probe 5}

\section{Saelborg skull}

Wood and peat overlying skull from Saelborg shore near Esbjerg (55 $30^{\prime} \mathrm{N}$ Lat, $8^{\circ} 27^{\prime} \mathrm{E}$ Long), Denmark. Coll. 1968 by Walter Wetzel, Geol.-Paläont. Inst., Univ. Kiel; subm. 1969 by Hermann Helmuth, now Univ. Peterborough//Toronto, Canada (cf. Wetzel and Helmuth, 1970). Comment: Libby age indicates wood must have been redeposited.

\section{KI-297. Saelborg, peat}

$$
\begin{array}{r}
2750 \pm 65 \\
800 \text { B.c. } \\
\delta C^{13}=-29.9 \%
\end{array}
$$

Sandy peat, overlying skull, dated by pollen analysis (F. R. Averdieck, Inst. f. Ur- und Frühgeschichte, Univ. Kiel) in Zone IXa (according to Overbeck, 1950). Comment (W.W.): Libby age is too young.

\section{KI-298. Saelborg, wood}

$14,890 \pm 160$ 12,940 в.C.

\section{Budrinna series}

Organic remains of burials from Budrinna, Libya $\left(26^{\circ} 42^{\prime} \mathrm{N}\right.$ Lat, $13^{\circ} 48^{\prime}$ E Long). Coll. and subm. 1970 by Helmut Ziegert, Seminar f. Vor- u. Frühgeschichte, Univ. Hamburg. Comment (H.Z.): samples are from different vaults of pre-Islamite burial place. Dates will help set chronology of pre-Islamite colonization of the Fezzan. Samples, except for KI-397, lay ca. 110 to $180 \mathrm{~cm}$ under surface (Ziegert, 1969; 1970). 
KI-392. Budr. A/6/Pr. 7

Carbonized remains of burial.

KI-393. Budr. A/5/Pr. 7

Carbonized remains of brain.

KI-394. Budr. A/9/Pr. 5

Carbonized remains of brain.

KI-395. Budr. A/8/Pr. 12

Carbonized remains of brain.

KI-396. Budr. A/7/Pr. 22

Carbonized remains of brain.

KI-397. Budr. A/11/Pr. $2+3$

Charcoal from fireplace above tomb, $45 \mathrm{~cm}$ under surface.

KI-398. Budr. A/11A/Pr. 11

Carbonized remains of brain.

KI-399. Budr. A/10D/Pr. 1

Twigs of tamarisc covering corpse in a vault.

KI-400. Budr. A/10B/Pr. 10

Carbonized remains of brain.

KI-401. Budr. A/10C/Pr. 7

Carbonized remains of brain.
$1360 \pm 60$

A.D. 590

$\delta C^{1.3}=-22.8 \%$

$$
\begin{array}{r}
1270 \pm 60 \\
\text { A.D. } 680 \\
\delta C^{13}=-24.2 \% 0
\end{array}
$$

$$
1470 \pm 110
$$
A.D. 480

$$
1450 \pm 55
$$

A.D. 500

$\delta C^{13}=-23.0 \%$

$$
\begin{aligned}
& 1330 \pm 40 \\
& \text { A.D. } 620 \\
& \delta C^{13}=-21.2 \%
\end{aligned}
$$

$$
1530 \pm 160
$$

A.D. 420

$\delta C^{13}=-25.6 \%$
$1290 \pm 45$
A.D. 660
$\delta C^{13}=-22.5 \%(c 0$

$$
1780 \pm 80
$$

A.D. 170

$\delta C^{13}=-23.9 \%$

$1840 \pm 150$
A.D. 110
$\delta C^{13}=-23.2 \%$

$1350 \pm 50$
A.D. 600
$\delta C^{13}=\cdots-21.3 \%$ 
KI-402. Budr. B/3/Pr. 2

Stalks of a mat underlying corpse in a vault tomb.

\section{KI-403. Budr. D/2/Pr. 7}

Garbonized remains of brain.

\section{KI-438. Las Tortolas}

$$
1690 \pm 110
$$

A.D. 260

$\delta C^{13}=-26.4 \%$

$$
1780 \pm 65
$$

A.D. 170

$\delta C^{13}=-27.88^{\prime}{ }^{\prime}$

$$
1050 \pm 90
$$

A.D. 900

$\delta C^{13}=-22.1 \%$

Sample of a wood pile on top of Las Tortolas, $6330 \mathrm{~m}$, Chile $\left(29^{\circ}\right.$ $51.0^{\prime} \mathrm{S}$ Lat, $69^{\circ} 53.5^{\prime} \mathrm{W}$ Long). Coll. 1971 and subm. by Klaus Krisch, Inst. f. Physiol. Chem., Univ. Kiel. Comment (K.K.): wood could have been piled during realm of Incas, for use in fire signals (summit of empire ca. A.D. 1400 to 1500 ).

\section{RELHFNCIS}

Degens, E. T., 1969, Biochemistry of stable isotopes, in: Folinton, G., Murphy, M. T. I. (eds.), Organic Geochemistry, Berlin, Springer Verlag, chap. 12, p. 304-399.

Enge, Cünter, 1971, $\mathrm{C}^{13}$ - und $\mathrm{C}^{11}$. Untersuchungen an rezenten Wasserproben: Staatsexamensarbeit, Kiel.

Klug, Heinz, 1968, Morphol. Studien auf den Kanarischen Inseln: Geog. Inst. Kiel Schr., v. 24 , p. 3.

Kählmorgen-Hille, G., 1963, Quantitative Untersuchungen der Bodenfauma in der Kieler Bucht und ihre jahreseitlichen Veränderungen: Kieler Meeresforschungen, v. 19 , p. 4 ? -66 .

Overbeck, Fritz, 1950, Dic Moore Niedersachsens: Bienen-Horn, Niedersächsisches Amt für Landesplanung und Statistik, p. 65-68.

Seibold, E. et al., 1971, Marine geology of Kiel Bay: sth internatl. sedimentological cong., Heidelberg, Germany, 1971; Sedimentology of parts of Central Europe, Guidebook, p. 209-285.

Suess, Erwin, 1971, Diagenesis of organic matter in Baltic Sea sediments: 8th internatl. sedimentological cong., Heidelberg, Germany, 1971, abs., p. 99.

Wezel, Walter and Helmuth, IIcrmann, 1970, Ein fossiles trepaniertes menschliches Stimbein: Naturw. Ver. Schlesw.-Holst. Schr., v. 40, p. 97-100.

Williams, P'. M.. Gordon, L. I., 1970, Carbon-13: Carbon-12 ratios in dissolved and particulate organic matter in the sea: Deep-Sca Rescarch, v. 17, p. 19-27.

Ziegert, Helmut, 1969, Uberblick zur jüngeren Besiedlungsgeschichte des Fezzan: Berliner Geog. Abh., no. 8, 1969 , p. 49-58.

1970, Human cultural development and climatic conditions in the Sahara during Pleistocene times: Internatl. conf. on African geol., Ibadan, Dec. 1970. 